\title{
Organism Substrain
}

National Cancer Institute

\section{Source}

National Cancer Institute. Organism Substrain. NCI Thesaurus. Code C117456.

A further sub-classification of the organism strain. 